\title{
Study on Application of Reliability Growth in the Development of Civil Aircraft
}

\author{
Zhang Junyi ${ }^{1, a, *}$ \\ ${ }^{1}$ Beijing Aeronautical Science \& Technology Research Institute of COMAC, Beijing, China \\ a hope_zhang@163.com \\ *corresponding author
}

Keywords: Civil Aircraft Development and Manufacture, Reliability Growth Test, Reliability Enhancement Test, High Accelerated Stress Screen.

Abstract: In the present paper, key tasks and working procedures in implementation of Reliability Growth Test techniques of HALT and HASS are identified, from their demands and current application status in research and development of commercial airplanes. Key technologies in Reliability Growth Test are also analyzed to provide support in testing methods for improvement on the design and manufacturing processes for on-board systems, in order to improve the quality of the system and increase its reliability, and then reduce the development lead time.

\section{Introduction}

With the rapid development of new technologies in civil aviation and the more and more comprehensive functions of airborne hardware equipment, the negative effects caused by hardware design errors and manufacturing defects are becoming more and more difficult to be controlled:

1) due to the size restriction and the application of a large number of new technologies, the modules are more complex and more integrated;

2) high mobility and wider range of routes lead to more severe environmental conditions in which the unit is located;

3) the capability of the multitasking platform leads to the diversity of the environmental features it is experiencing;

4) reliability life expectancy is high, and some can reach tens of years or even longer.

The high reliability requirements of the system lead to very high reliability requirements of each component unit, and the development cost will be increased by $25 \%-150 \%$. At the same time, because the design or manufacturing process is not perfect, the resulted increase in maintenance cost is considerable. As shown in Table 1, the relevant design correction or maintenance costs vary with the exposure time of the defect in the aircraft component. The earlier the latent defects are discovered before the trial stage, the lower the cost for the design correction. And after the mass production with increased production output, the costs for return to factory correction of the defects will be increased exponentially (Table 1). Therefore, research on the reliability growth technology for complex systems based on the development phase of large civil aircraft is in line with national strategic requirements, and it is important in the establishment of "made in China" brand.

Table 1. Costs of fault correction for an electronic product at all stages of life cycle

\begin{tabular}{|c|c|}
\hline $\begin{array}{c}\text { Time when the latent defect is } \\
\text { identified }\end{array}$ & Defect correction costs \\
\hline Design and development phase & $\$ 35$ \\
\hline Before the design is finalized & $\$ 175$ \\
\hline Pre-production trial phase & $\$ 368$ \\
\hline Batch production stage & $\$ 17000$ \\
\hline Usage phase & $\$ 690000$ \\
\hline
\end{tabular}


To increase the efficiancy of the traditional simulation test to ensure product reliability, in 1967, the Roma airshow center in the United States proposed the accelerated life test method (Accelerated Life Testing:ALT), and in 1988 G.K.Hobbs in the United States proposed a highly accelerated life test (Highly Accelerated Life Testing:HALT) and highly accelerated stress screening (Highly Accelerated Stress Screening:HASS). These three kinds of testing methods together with the accelerated degradation test (Accelerated Degradation Testing:ADT) constitute the accelerated reliability testing technology [1]. HALT and HASS are also known as the provocation test, in which the accelerated stress is used to effectively stimulate the latent defects of specimen, the corresponding improvement strategies are established through the defect location and fault mechanism analysis etc., to achieve speedy reliability improvements. HALT is mainly concerned with the design process and in Boeing it is called reliability enhancement test technology (Reliability Enhancement Test:RET) when it is applied, while HASS is mainly aimed at the production process.

\section{Current status of reliability growth test method in aviation industry applications}

In the international civil aircraft airborne electronic products research and development, highly accelerated life test HALT and highly accelerated stress screening method HASS have been widely used. There are a number of highly influential reliability growth test guidelines and specifications: RET for the fault prevention strategy in D6-57067 from Boeing, HALT operating procedures EAD460/12/00 issued by EADS and Airbus Company in 2001, and HALT Guidelines and HASS Guidelines from the U.S. company QualMark. As specified in D6-57067, RET is used to eliminate design and manufacturing defects in Boeing airborne equipment before it enter into production, RET must be performed on all new equipments, in-service low reliability equipments and existing equipments to be overhauled and to be re-evaluated, to eliminate the design defects and to reduce costs in their life cycles.

In the aviation application, RET must be carried out on new development products as defined in Boeing company procedures. In 1994, intensive tests of RET and ALT (accelerated life test) were carried out on 40 Boeing 777 line replaceable components (Line, Replaceable Unit, LRU), and compared with the conventional service station failure number were collected, RET excitation of fault is evident.

In addition, 1000 cycles of replacement maintenance tests were performed on 390 sets of LRU (replaceable unit) in the Boeing 777 aircraft. Among 217 sets of LRU RET tested, the replacement rate was 4\%, and in the remaining 173 sets of LRU with no RET, the replacement rate was 35\%. It can be seen that RET can greatly reduce the maintenance replacement rate and maintenance costs.

RET is also a supplement to the current comprehensive environment stress reliability acceptance test technology. The method is from the Boeing company successful engineering example of airborne electronic equipment acceptance check, i.e.: first, enhanced comprehensive environmental stress screening is used to reduce the fault exposure time, then, the reliability index of the airborne electronic equipment is accessed quantitatively by other engineering methods or statistical techniques. In the past few years, this method has been verified in the reliability acceptance test of dozens of airborne electronic equipment batch production. The feasibility and effectiveness of this method have been verified. [5]. RET and RQT (reliability identification test) were carried out on 4 types of equipments in Boeing 777 aircraft, and RQT was performed on RET improved 4 types of equipments, the comparison of the number of faults is given in Figure 1. It can be seen that the effect of RET excitation fault is very significant, and it is helpful for the acceptance check of airborne electronic equipments. 


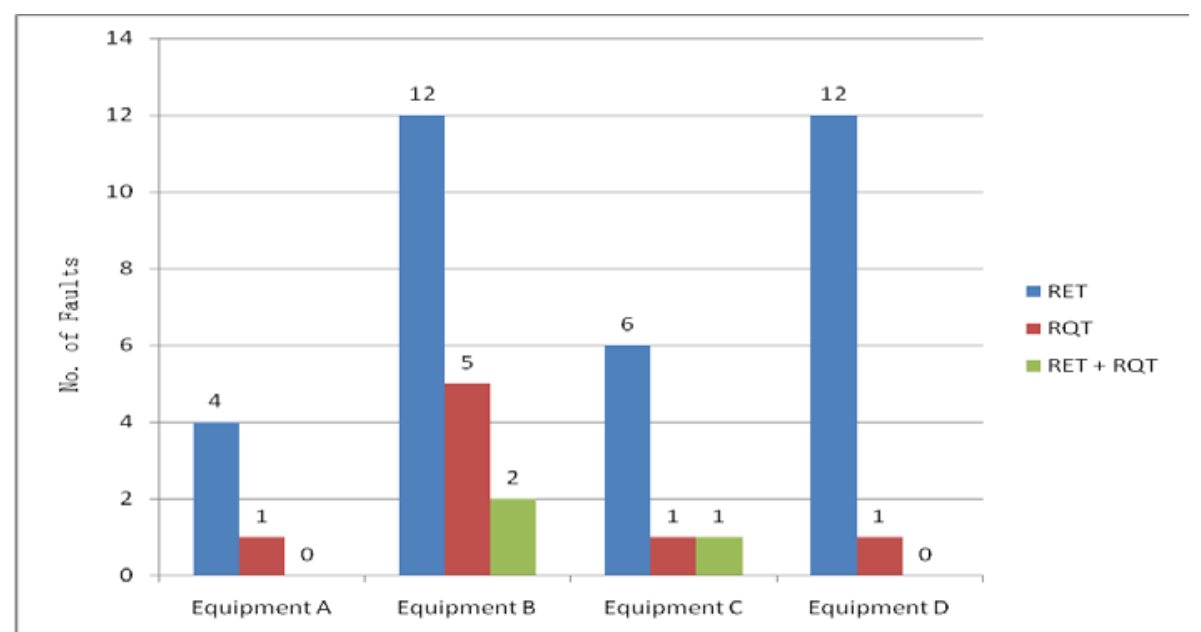

Figure 1. Comparison of the number of faults in RET and RQT

It can be seen from the application of the Boeing company, the reliability enhancement test is a very effective means for the rapid excitation of product defects. In the development stage, the enhancement test can greatly reduce defects after the product is service, so as to reduce the maintenance cost and gain significant economic benefit.

With the rapid development of the civil aviation industry in China, further research and development on this technology is enhanced. In the development of a certain type of C919 airborne electronic products, there have been some practice runs of HALT and HASS test methods. Design and process defects can be found highly efficiently through the HALT test and can be addressed accordingly. At the same time, highly accelerated stress screening test using the new HASS test method was carried out on the products before delivery according to the previously defined profiles. Those products were successfully passed the screening test and ATP test, and delivered to customers. The screening efficiency was increased more than 10 times. The application of this concept and test method has improved the reliability level of the product, greatly improved the R \& D efficiency and reduced the R \& D cycle time.

\section{Reliability Growth Test Implementation Process}

HALT or HASS, as a method of excitation test, is based on the theory of fault physics. It regards faulty or failure as the main object of study, and improves the reliability by stimulating, researching and solving the product defects. Figure 2 illustrates how HALT and HASS are integrated into aircraft design, development, and manufacturing processes.

Design Development,AirwathinessCatification BatchProducion

Product

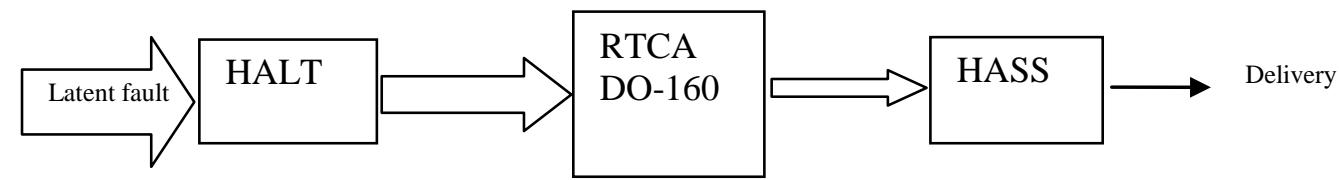

Figure 2. Relationship between reliability growth test and aircraft development cycle.

\subsection{Failure modes and effects analysis: FMEA}

In the aircraft design process, through the analysis of the aircraft components of various kinds of latent failure modes and their effects on aircraft grade function, and each latent failure modes are classified according to the severity, put forward prevention measures, in order to improve the safety and reliability of the aircraft.

Before the reliability growth testing, FMEA can be used to analyze the existing design from theoretic point of view to find in the design of potential weak links, to strengthen the pertinence of the testing; to analyze the environmental stress which affects product reliability, so as to determine the best incentive stress for the reliability growth test; to determine the fault test method in 
reliability growth test according to its possible failure mode; to establish the internal relationship between faulty mode and failure mechanism, in order to carry out effective faulty analysis after the growth test; to obtain product faulty modes that may occur in the reliability growth test, and to give the improvement scheme to contribute to a better analysis of reliability growth test results and put forward the improvement measures.

\subsection{Sensitive Stress Analysis}

Sensitive stress analysis is a kind of qualitative analysis to determine the influence of environmental factors on the reliability of the system. Through the analysis of every environmental stresses which will affect the reliability of product and its consisting units, all environmental stresses are classified according to the extent of their influences on products and consisting units, to identify those most influential sensitive stresses on product reliability according to the classification results, and then reliability growth tests are carried out selectively on products to effectively and rapidly stimulate the latent defects, which is based on the fact that the environment for the full life cycle of products has limited effect on excitation of latent system defects.

From the environmental stress screening, based on limited screening test results on 13 stresses with certain representativeness and among them the random vibration and temperature cycling are the most effective incentive stresses. According to related experiments, it can be concluded that for most electronic products, the full axis random vibration environment and temperature cycle with large temperature variation rate will be the most effective excitation stresses. However, this conclusion can not be absolutization: a new sensitive stress can be generated for a specific product and a specific working environment. Therefore, the introduction of stress sensitive analysis into the basic process of reliability growth test is essential and has important guiding significance to the profile design for the reliability growth test. Environmental stresses with higher influence on reliability are selected through the sensitive stress analysis to more efficiently excitate latent defects of products.

\subsection{Design of test scheme}

Using the reliability growth test to discover and identify the specimen failure is largely dependent on the degree of sensitivity and the degree of perfection in the fault detection. Therefore, it is necessary to practically analyze performance parameters that can best represent the characteristics of the product, and to draw up appropriate test plan. The test plan should include test signal set, measurement point distribution, test method, test time interval and so on.

There is no fundamental difference between reliability growth test design in principle and other reliability tests, but there is a need to pay special attention to the analysis of fault location and mechanism, to achieve optimal design of fault location. However, the reliability growth test can not introduce overly complex test schemes for fault location, and there is also a trade off between test coverage and test cost. Therefore, it is necessary to find out the test set which can reflect the main failure modes on the basis of FMEA, and realize the detection and location of the sample fault with the minimum test cost. The selection of a test set can generally follow the following principles:

1) make full use of FMEA information, be able to sensitively reflect the main faults of the test piece;

2) pay attention to test simplification, so that the test parameters can not only reflect the fault condition of the test piece, but also facilitate the test;

3) focus on testing the main performance indicators and main functional modes.

\subsection{Testing Procedure}

In the testing procedure, it mainly follows the "step by step" principle, in accordance with the test stress for the incentive strength of specimens from low and high loading. Therefore, it is necessary to arrange the proper loading order and step stress. Such test arrangement can expose gradually the fault in the test piece during the test, which is convenient for fault locating and analysis. The most commonly used test steps are cooling temperature stepped stress test, heating 
temperature stepped stress test, high temperature variable rate temperature cycle test, full axis random vibration stepped stress test and comprehensive environment test.

\subsection{Test Profile Design}

The reliability growth test profile is a description of stress loading sequence for reliability growth tests. It is the relationship diagram between stress parameters and time for testing directly. The design of the test profile has important influence on the validity of reliability growth test. In the design of reliability growth test profiles, special attentions should be paid to the following:

1) force magnitude settings

For the same type of excitation stress, the greater the force magnitude, the higher the excitation efficiency of the product fault, and the shorter the test time. However, the highest stress magnitude settings must not change the failure mechanism of the test, i.e. fault excitated in the reliability growth test must be able to be reproduced in actual use, which makes the targeted optimization design of practical significance. The excitation stress in the test can not be infinite due to the limitation of the capacity of the test equipment. The greater the strength level, the higher the test energy consumption.

2) optimization of stress combinations

Combinations of various stresses should take into account of the actual conditions of the product and cannot be just simply assembled. For example, humidity and high change rate temperature cycle cannot be used at the same time, because in the condition of high temperature change rate, fluid (water vapor) change in three states will become extraordinary which is in servere discrepancies with the products of the actual working environment. The fault excitated will lose its practical significance. Generally, full axis random vibration stress has the highest excitation efficiency for latent defects, followed by temperature cyclic stress. The baseline scheme generally uses a combination of full axis random vibration and high temperature variable rate temperature cycles.

\subsection{Validation of the effectiveness of the optimization design}

For the fault detected in the experiment, it is necessary to carry out on-site analysis of the fault in reliability growth test requirements. Relevant measures of optimization design will be established based on understanding of the failure mechanism, and on-site repair will be carried out accordingly. And then, after the implementation of optimization measures the specimen will be tested again to confirm the validity of the optimization measures. The test will proceed to higher stress levels to find further new faults. The effectiveness of the design optimization includes two aspects: whether the current fault is removed and if new fault is found.

In addition, it should also be considered in the reliability growth test the cost-effectiveness in optimization design, in which optimization measures will not apply to all faults, but it will take into consideration of the cost and benefit of improved design. And in some cases their feasibilities will also be considered.

\section{Outlook of reliability growth test technology}

The application field of reliability growth test technology is relatively sensitive and currently we have only very limited access to the core technologies. All we have seen are in the engineering application of this technology, and the open test platform and other aspects, focusing on commercial publicity.

Generally speaking, in the field of civil aircraft, the research on reliability growth of new products is confronted with the following problems:

1) Currently, the test stress and loading order etc. are based on guidelines from overseas industries. Test objects, test profiles and test plan design are established in lack of scientific and systematic theoretical basis, and an authoritative standard; 
2) Lack of knowledge in excitation mechanisms. The growth mechanism of super Gauss random vibration test has been studied in-depth, but the consistency in the test object failure mechanism and vibration acceleration model also needs further investigation;

3) Civil aircraft development should be closely focused on the airworthiness certification. It is not clear at the present stage the implementation of time and objectives of the reliability growth test.

\section{References}

[1]. JIANG Pei, CHEN Xun, ZHANG Chun-hua, TAO Jun-yong.Reliability enhancement testing. Structure \& Environment Engineering[J ]. 2003,30(1).

[2]. Silverman M. Summary of HALT and HASS results at an acceleratedreliability test center [A]. In : 1998 Proceeding Annual Reliability and Maintainability Symposium[C] , 1998.

[3]. Xu Jinfei. Aviation airborne electronic equipment reliability enhancement test method of [J]. China science and technology information, 2012, (11).

[4]. Ke Xianmeng, Xu Chenguang. Study of Aircraft test and screening test method for avionics reliability. Fifth International forum on civilian aircraft avionics systems, Shanghai, 2016.

[5]. Zhu Weihua, Chen Ji, Tao Junyong, Zhang Chunhua, Jiang Pei. High accelerated life test (HALT) and stress screening (HASS), [J], Strength and Environment,2012,29(4).

[6]. Cao Shunan, Li Ping. Application of reliability enhancement test in acceptance test of airborne equipment [J]. reliability and environment test of electronic products, 2016, 34(1). 